\title{
Urgences
}

\section{Le verbe se fait chair : quelques modifications signifiantes de l'imparfait à l'indicatif présent dans le manuscrit du Salut de l'Irlande de Jacques Ferron}

Jean R. Côté

Numéro 24, juillet 1989

Le manuscrit sous l'angle

URI : https://id.erudit.org/iderudit/025535ar

DOI : https://doi.org/10.7202/025535ar

Aller au sommaire du numéro

Éditeur(s)

Urgences

ISSN

0226-9554 (imprimé)

1927-3924 (numérique)

Découvrir la revue

Citer cet article

Côté, J. R. (1989). Le verbe se fait chair : quelques modifications signifiantes de

l'imparfait à l'indicatif présent dans le manuscrit du Salut de l'Irlande de

Jacques Ferron. Urgences, (24), 89-93. https://doi.org/10.7202/025535ar d'utilisation que vous pouvez consulter en ligne. 


\section{Le verbe se fait chair: quelques modifications signifiantes de l'imparfait à l'indicatif présent dans le manuscrit du Salut de I'Irlande de Jacques Ferron Jean R. Cóté, Université d'Athabaska}

Le problème du temps du récit et des temps des verbes est un, mais non le moindre, des divers problèmes de narratologie auxquels est confronté l'écrivain au cours de la tabrication d'un texte de fiction. Les trente et quelques modifications apportées aux temps des verbes dans le manuscrit du Salut de I'Irlande de Jacques Ferron trahissent la préoccupation et le travail de l'auteur à cet égard. Une série de treize modifications de l'imparfait à l'indicatif présent en pages 47 et 48 a spécialement attiré notre attention. En scrutant sous ces indices, en apparence banalement grammaticaux, nous avons mis à jour les signes certains d'un projet esthétique global dont l'hermétisme du texte imprimé ne laissait guère entrevoir que de bien minces ficelles. Mais d'abord, un coup d'œil au passage en question.

La première partie est immédiatement écrite à l'indicatif présent dit "historique": "C'est une fille de la campagne..." suivi de: «Elle remplace de son mieux... à mesure qu'elle vieillit." Plus loin, il se produit comme un fléchissement dans cet imparfait: "C'est un luxe que CDA Haffigan ne POUVAIT pas se payer." (réécrit en bleu POURRAIT, correction qui a pour effet de présentifier l'énoncé). Et aussi: «lequel ÉTAIT (raturé, suscrit en bleu EST) avec la bagosse..." "Telle ÉTAIT (raturé) EST..." etc.

Après cette première mention du père, le récit revient à la présentation de la mère: "Cette sincérité, M'man la lui fournISSAIT (raturé) IT. D'honnêteté: M'man ÉTAIT (raturé) EST honnête pour deux, de courage et d'amour pour le travail: M'man en AVAIT (raturé) A de reste. Elle lui ÉTAIT (raturé) EST indispensable." 1 Ainsi de suite, toute une série de modifications qui ne peuvent passer inaperçues, d'autant plus que, bleues sur

1 Manuscrit du Salut de I'Irlande, Longueuil, automne 1970, p. 4748. A l'imprimé, Le salut de l'Irlande, Montréal, Éditions du Jour, 1970, pp. 65-66. 
un texte rouge, elles sont groupées dans une seule page. Elles n'ont pu que susciter l'analyse de la grammaire particulière des temps des verbes qu'elles contribuent à articuler, une grammaire comportant une signification complémentaire à la simple marque de rapport temporel.

Quelques mots maintenant sur la problématique que ce réseau de modifications manuscriptiques a soulevée. Ce n'est que bien plus tard, après la présentation de la mère de Connie, le narrateur, que le récit relate sa mort, épisode capital celui-là puisqu'il est le déclencheur de l'initiation de Connie et de son engagement à "sauver l'Irlande". Mais jusqu'à ce moment-là, à cause de l'indicatif présent, la mère "est" vivante, "est" représentée comme actuelle dans le récit. Elle vit hic et nunc avec ses humeurs, ses gestes, ses projets...

La présentation de la mère est donc, avant modification, écrite à l'imparfait. Mais cet imparfait en arrive à jouer, selon la connotation que l'on sait à l'usage ordinaire de la langue qui tend à parler de quelqu'un au passé après son décès, un rôle dans la matière même de l'œuvre. En effet, plus tard dans le récit, comme on l'a dit, la mère mourra. L'imparfait de la première version comporte donc, étant donné la nature même de la diégèse, une surcharge de signification, surcharge qui vient infirmer le caractère a-temporel qu'on alloue à l'imparfait, depuis Käte Hamburger, dans une fiction narrative. ${ }^{2}$ Cependant, l'annonce de cette mort sous forme du récit des funérailles, très justement écrit à l'imparfait celui-là, ne doit survenir que plus tard, au neuvième et dernier chapitre. Ferron revient donc réécrire au présent le passage où Connie nous décrit sa mère. Sans doute le fait-il, davantage que pour des raisons de cohérence avec les quelques verbes au présent du début du paragraphe ${ }^{3}$, pour des raisons de

2 \&ll est désormais clair que ni la présentation au prétérit ni celle au présent ne suscite l'expérience du passé. [...] L'interprétation temporelle est invalidée par le simple fait que l'imparfait n'indique pas non plus le passé. II reste à se demander pourquoi le présent n'a pas une fonction d'actualisation fictionnelle qui dépasse celle de l'imparfait.» Käte Hamburger, Logique des genres littéraires, traduit de l'allemand par Pierre Cadiot, Paris, Éditions du Seuil, 1986, p. 105.

3 Depuis «ll faut maintenant que je parle de M'man [...[ elle VIENT de Saint-Wenceslas $*$, page 46 , jusqu'à ${ }^{\prime}$ C'est un luxe...», indicatifs présents écrits à la première version ceux-là. Ce qui n'infirme en rien que ce sont les ratures et modifications qui ont attiré notre attention sur 
tension romanesque, d'articulation des multiples et complexes sous-récits et d'organisation des éléments textuels en vue du projet esthétique global. C'est peut-être plus précisément dans le but de ménager une accentuation de dernière instance, car c'est en finale que surviennent et la mort de la mère et le point fort de l'initiation de Connie. On peut à bon droit supposer que la possibilité ou la nécessité de cette accentuation, de cette réunion en faisceaux des éléments du récit, s'est imposée à l'esprit de Ferron dans le cours de la rédaction des chapitres qui suivent celui de la présentation de la mère, comme peut en témoigner la polychromie des encres employées.

En effet, si l'on examine la séquence rédactionnelle des modifications des temps de verbes que les couleurs d'encre nous permettent de supposer, on aura une première série (pages 30 et 35) faite à l'encre bleue sur texte en feutre orange, la longue série de treize (pages $47-48$ ) à l'encre bleue sur un texte en feutre rouge, deux autres (p. 69 et 70 ) à l'encre bleue sur encre bleue et une dernière série de trois à la fin du dernier chapitre, en feutre rouge sur feutre rouge. Cette séquence suggère, les premières corrections ayant été faites si l'on se base sur l'analogie couleurs, en même temps que les corrections ultérieures (sauf naturellement les trois dernières), que Ferron était de plus en plus attentif à l'usage qu'il faisait du temps des verbes à mesure que s'élaborait son projet fictionnel et que c'est en rédigeant ses chapitres ultérieurs qu'il revenait modifier ses temps des premiers chapitres. Elle pourrait tout aussi bien nous laisser entendre, cette séquence, qu'à une maîtrise de l'outil grammatical a succédé une systématisation de son usage, jusqu'à ce que cet outil devienne une scène discursive digne d'être porteuse de l'idéologie du devenir et de la rédemption. Un inventaire des nombreux autres jeux de temps de verbes relevés au texte imprimé, mais que le manuscrit ne trahit pas nécessairement, pourrait ici faire foi de cette systématisation.

Mais revenons à notre exemple. Comme on le sait, dans d'autres contes de Ferron, la mort est très souvent signifiée dans et par le passage d'un JE à un IL narrateur. La mort de la mère de Connie dans Le salut de l'Irlande, en fidélité à l'esprit

la particularité du procédé narratif et que ce dernier trouve son sens dans l'ensemble contextuel. 
des singulières connotations de la grammaire ferronnienne, est cette fois signifiée par le simple appareil linguistique du passage du présent à l'imparfait. Ou plutôt, c'est l'état de la mère de Connie encore vivante qui est signifié par des indicatifs présents qui, tels que dénoncés par les modifications au manuscrit, sont en fait des retouches dans le contexte d'un ensemble narratif à l'imparfait, lequel temps de verbe, soulignons-le, s'inscrit en référence à un temps de narration autobiographique ultérieur à la mort du personnage. En réécrivant le passage des pages 47 et 48 à l'indicatif présent, passage qu'il aurait pu en toute logique narrative laisser à l'imparfait, Ferron joue donc à préserver le secret de la mort ultérieure de la mère. Ou mieux, il veut nous réserver pour plus tard la révélation de cette mort, "enfirouapette" textuelle digne d'un maître-conteur au fait de son auditoire. 4

On constate donc que ces modifications de temps de verbes, à première vue banales et ponctuelles, n'en sont pas moins conséquentes à longue portée sur l'ensemble du récit. Par elles, Ferron non seulement donne vie à un personnage de la diégèse, mais, à un tout autre degré, effectue aussi du travail de création par une sorte de sémiotique du style. Car ce qui peut paraître, à première vue, comme une simple modification de grammaire ou de style s'avère aussi, en profondeur, participer de la signifiance de l'ensemble: la fiction narrative doit présenter M'man comme bien vivante, par des verbes au présent, pour utiliser l'impact dramatique maximum de cette mort en dernier chapitre. En somme, les temps des verbes font préalablement mieux vivre la mère, pour rendre encore plus actuel et actantiel l'avènement de sa mort, ou si l'on veut, pour la faire mieux mourir. Mimesis stylistique cruellement nécessaire, sacrifice ultime à une esthétique de la création littéralement verbale qui, en concordance avec l'esprit du conte, tisse insidieusement son filet et ménage ses effets pour le punch final.

On pourrait objecter la simplicité du procédé à cause de sa connotation à l'énoncé de réalité que l'on sait. Mais l'invention véritable et véritablement humaine ne réside-t-elle pas

4 «Tout l'art du roman repose sur la crédulité du lecteur, ce que le conteur populaire sait très bien: il ne ment pas, il trompe, et il trompe pour s'adresser à mots couverts à des non-initiés. „ Jacques Ferron, «a part du grimoire», Du fond de mon arrière-cuisine, Montréal, Editions du Jour, 1973, p. 46. 
justement dans de nouvelles et harmonieuses combinaisons d'éléments tirés de la langue ordinaire? Ainsi, pour pénétrer le sens véritable de cette invention, ne devrait-on pas plutôt se pencher sur la symétrie se dessinant entre la signifiance des indicatifs présents de notre exemple et la signifiance des imparfaits de leur entourage textuel et en particulier de ceux du dernier chapitre? On verra que cette symétrie vient renforcer en langage grammatical le caractère oxymoriquement concordant de la mort de la mère et du rite initiatique du fils, seule naissance qui soit véritable, seule mort qui ait un sens. La voie tracée par cette interprétation nous amènera inévitablement à conclure que la substance du Salut de l'Irlande, la genèse de l'homme libre, vient à germination, comme on l'a vu, dans le cours même de la genèse du récit. Et que ce récit, grimoiremiroir où l'auteur se crée en écrivant et où le lecteur par empathie peut se voir, n'est rien d'autre qu'un verbe signifiant parmi les innombrables verbes d'un Grand CEuvre d'où jaillissent tous les espoirs de la naissance et du devenir. Le manuscrit du Salut nous convie donc à cette "composition en abîme" que Jean Marcel a déjà si admirablement perçue comme essentielle à l'œuvre de Ferron. Ainsi, notre compte rendu d'analyse, en abîme tout autant, n'en serait-il qu'un modeste miroitement.

5 A propos d'autres éléments textuels, mais toujours sous le même rapport, Jean Marcel écrit: $\propto$ Tous les personnages des contes de Ferron sont un peu conteurs eux-mêmes, et cette sorte de composition en abîme, à la façon des peintres flamands, est une tentative d'absorption du contenu par sa propre signification.» Jacques Ferron malgré lui, coll. «Frères chasseurs», Montréal, Parti pris, 1978, p. 49. 